Provided for non-commercial research and education use. Not for reproduction, distribution or commercial use.

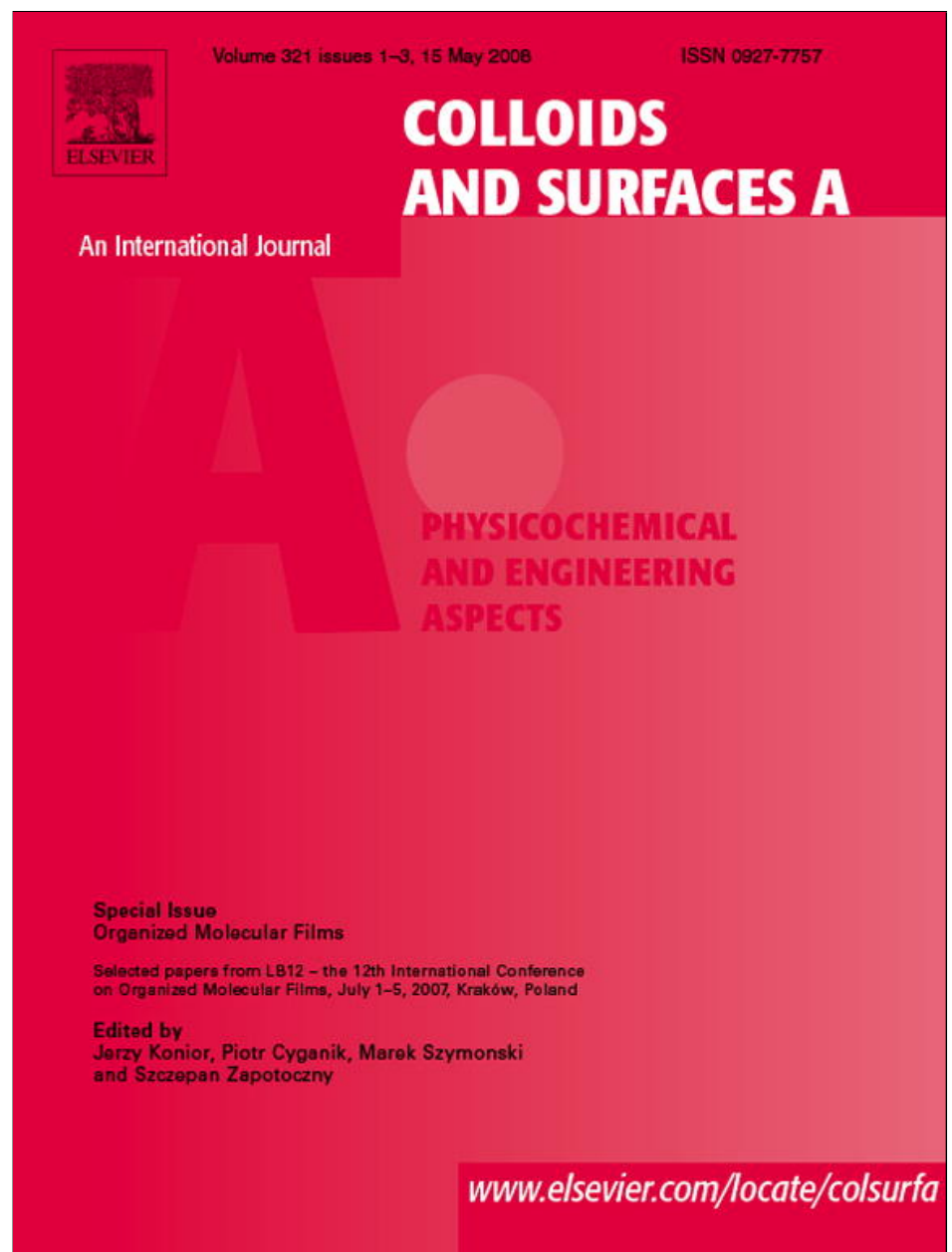

This article appeared in a journal published by Elsevier. The attached copy is furnished to the author for internal non-commercial research and education use, including for instruction at the authors institution and sharing with colleagues.

Other uses, including reproduction and distribution, or selling or licensing copies, or posting to personal, institutional or third party websites are prohibited.

In most cases authors are permitted to post their version of the article (e.g. in Word or Tex form) to their personal website or institutional repository. Authors requiring further information regarding Elsevier's archiving and manuscript policies are encouraged to visit:

http://www.elsevier.com/copyright 


\title{
Template-assisted self-assembly of colloidal crystals
}

\author{
P. Huber ${ }^{\mathrm{a}, *}$, T. Blättler $^{\mathrm{b}}$, M. Textor ${ }^{\mathrm{b}}$, W. Leitenberger ${ }^{\mathrm{c}}$, \\ U. Pietsch ${ }^{\mathrm{d}}$, T. Geue ${ }^{\mathrm{a}}$ \\ ${ }^{a}$ Laboratory for Neutron Scattering, ETH Zurich \& PSI, 5232 Villigen PSI, Switzerland \\ ${ }^{\mathrm{b}}$ Laboratory for Surface Science and Technology, Department of Material Science, \\ ETH Zurich, 8093 Zurich, Switzerland \\ ${ }^{\mathrm{c}}$ Institute of Physics, University of Potsdam, D-14415 Potsdam, Germany \\ ${ }^{\mathrm{d}}$ University of Siegen, Solid State Physics Group, D-57068 Siegen, Germany
}

Received 10 July 2007; received in revised form 15 November 2007; accepted 17 January 2008 Available online 31 January 2008

\begin{abstract}
We present the self-assembly of polystyrene particles to colloidal crystals via the dipping method on a topologically structured template. On this system we demonstrate the potential of grazing incidence small angle X-ray scattering as an analytical tool to monitor properties of colloidal crystals. Moreover, energy dispersive X-ray reflectometry has been used to investigate the dynamics of the self-assembly in situ.

(C) 2008 Elsevier B.V. All rights reserved.

Keywords: Colloidal crystal; Template-assisted self-assembly; Azo-polymer; GISAXS; EDXR
\end{abstract}

\section{Introduction}

Micro-structured materials for photonic applications attract currently a worldwide interest and a large effort is put in their development. A majority of projects is dedicated to photonic crystals. These are materials with a periodically varying dielectric constant scattering light such that the interfering electromagnetic waves open up a photonic band gap, i.e. a region in the electromagnetic spectrum, where propagation of light is stopped [1]. The commercialization of photonic crystals is yet far from being achieved because the creation of photonic devices with well-defined properties is still very difficult and not really reproducible.

A versatile approach towards three-dimensional photonic crystals relies on the inversion of colloidal crystals [2,3]. The interspaces of the colloidal crystals are filled with high refractive index materials (e.g. polycrystalline titan dioxide or silica) in a first step and thereafter the colloids are dissolved. The particle assemblies act as templates only, they do not have to supply the high difference in refractive index which is necessary for the creation of a complete photonic band gap. Therefore, one

\footnotetext{
* Corresponding author. Tel.: +41 56310 5358; fax: +41 563102939.

E-mail address: patrick.huber@psi.ch (P. Huber).
}

can abandon the tight restrictions concerning the basis material for photonic crystals and polymeric material can be used for the particle assemblies. The crystals themselves are made up of highly monodisperse spherical colloids arranged in a crystalline structure that will form by self-assembly under proper conditions. This might lead to an efficient way of producing photonic crystals.

At present, most characterizations on colloidal crystals have been done by scanning electron microscopy (SEM) [3-5]. This allows imaging the top layer of a colloidal assembly. However, one does neither get any bulk information unless cleaving the crystal nor are in situ measurements on the formation of a three-dimensional assembly possible. Recently, small angle Xray scattering (SAXS) measurements have been performed on colloidal crystals [6]. This method provides information on the bulk material but the obtained structural information is averaged over the whole sample.

In the presented work we apply X-ray scattering techniques on colloidal crystals prepared by template-assisted self-assembly. Grazing incidence small angle X-ray scattering (GISAXS) [7] yields unique information about colloidal assemblies. In case of three-dimensional colloidal crystals the technique provides depth dependent information on the colloidal arrangement, i.e. the lattice type, domain size and the defect density over large areas. GISAXS is with no doubt a 

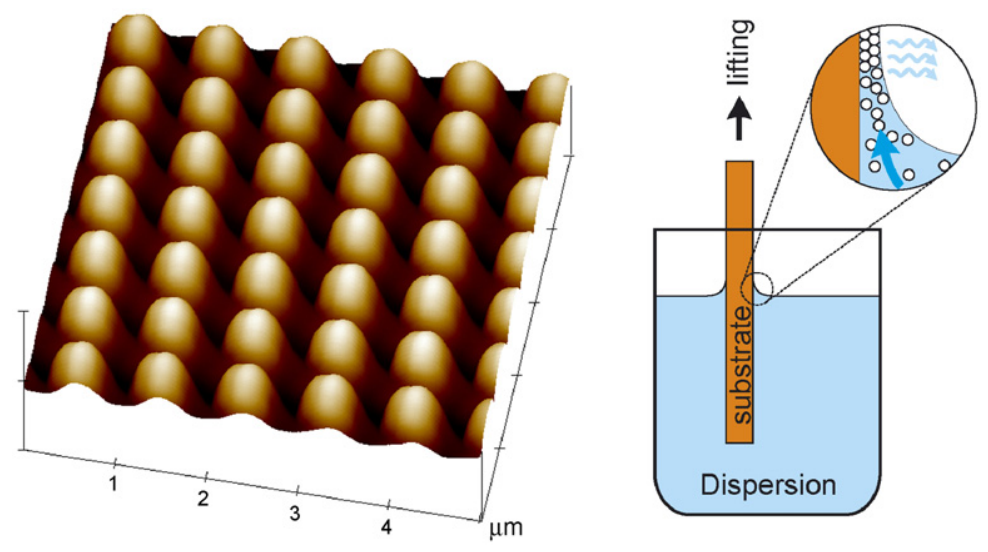

Fig. 1. Left: AFM picture of a surface relief grating formed by an azo-polymer. It has been cross-irradiated with an interference pattern that had a period of about $770 \mathrm{~nm}$. Right: The pre-structured substrate is dipped into the colloidal dispersion and slowly withdrawn.

valuable add-on to the characterization techniques such as SEM or SAXS. As an additional scattering technique, energy dispersive X-ray reflectometry (EDXR) [8] is used both to probe the collective dynamics of the particles leading to ordered structures during the self-assembly and the dynamics of the succeeding drying. EDXR combines an incident beam that consists of a whole spectrum of wavelength with an energy dispersive detector. Consequently, the technique allows probing different length-scales with a single measurement. It is therefore well suited for the investigation of dynamic processes requiring time-resolution.

\section{Experimental}

\subsection{Template preparation and colloidal assembly}

We prepared colloidal crystals through template-assisted selfassembly for the scattering experiments. The template consisted of an azo-polymer which was illuminated by an interference pattern of a laser at a wavelength close to the absorption maximum of the polymer [9], see Fig. 1. Due to the illumination, the polymer formed a surface grating. This is a one step process, i.e. no additional processing steps are needed after illumination. To ensure homogeneity of the polymer template several areas within the illuminated grating were inspected by AFM. Inhomogeneities amount to $20 \%$ in height and $1 \%$ in periodicity. If the grating period is properly matched with the diameter of the colloids, a long-range three-dimensional order of the colloidal assembly is realized [10]. For the self-assembly we used the dipping method, see Fig. 1, that yields assemblies with micrometer size [11].

For our samples we used negatively charged polystyrene particles provided by MicroParticles $\mathrm{GmbH}$ (Berlin) with diameters of 1028 and $760 \mathrm{~nm}$ (polydispersity 0.03 ). The period of the gratings was measured with AFM (Nanoscope DI 3100). Only gratings with periods that deviated less than $10 \%$ from the ideal value were used as templates. According to Allard et al. [10] this corresponds to the variance that will still give an almost defect free particle assembly. The grating heights amplitude varied for different gratings between 70 and $200 \mathrm{~nm}$.

\subsection{GISAXS on ordered colloidal assemblies}

The GISAXS measurements were performed at the beamline BW2 at HASYLAB, DESY in Hamburg [12]. Measurements were performed at 8.1 and $10 \mathrm{keV}$ photon energy using a defocused beam. A Bruker Smart Apex2 CCD-Camera System was used to collect the scattering intensities. The camera was positioned about one meter away from the sample with an evacuated flight tube in between. We choose scattering angles between $0.4^{\circ}$ and $0.8^{\circ}$.

\subsection{EDXR during self-assembly}

The EDXR experiments were performed at the bending magnet beamline EDR/D-13-2 at BESSY II in Berlin [13]. We worked at a slightly off-specular setup with an incident scattering angle of $0.3^{\circ}$ and an exit angle of $0.43^{\circ}$. The combination of the white X-ray emission spectrum between around 4 and $30 \mathrm{keV}$ and a silicon drift-diode as energy dispersive detector provided good counting statistics in a range of $-21.7 \times 10^{-5} \AA^{-1}<q_{X}<-2.9 \times 10^{-5} \AA^{-1}$ and $0.025 \AA^{-1}<q_{Z}<0.191 \AA^{-1}$. $q_{Z}$ is the direction perpendicular to the surface of the probe while $q_{X}$ lies in the scattering plane parallel to the surface. This setup provides a temporal resolution of $30 \mathrm{~s}$ per spectrum.

A droplet of colloidal dispersion was put on a topologically structured sample having a period matched to the diameter of the particles. The specimen was then placed into a sample environment on the beamline and the experiment was started. The sample environment allowed us to control the temperature and consequently the evaporation speed of the solvent.

\section{Results and discussion}

\subsection{Colloidal assembly}

Fig. 2 shows a light microscope image of a surface relief grating covered by a colloidal monolayer using the dipping method. The largest monolayers obtained so far exhibited domain sizes with areas of more than $300 \mu \mathrm{m} \times 300 \mu \mathrm{m}$. Only 


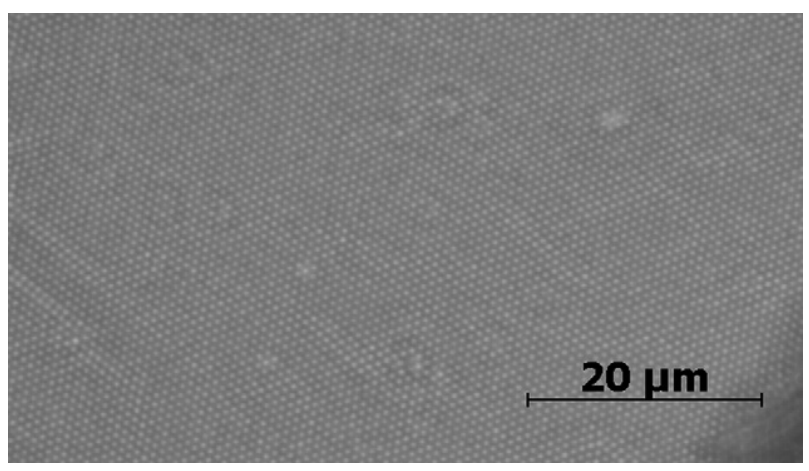

Fig. 2. Colloidal monolayer on top of a topologically structured azobenzene containing polymer film. The image was taken by a light microscope. The diameter of the colloids is $760 \mathrm{~nm}$, the grid spacing $664 \mathrm{~nm}$ and the averaged grid height is $100 \mathrm{~nm}$ measured by AFM

hair-line cracks are visible originating probably from the drying process [14]. We investigated the monolayer at different positions. Remarkably, the orientation of the probed parts of the colloidal monolayer does not change. This is rare for samples on flat substrates indicating that the surface grating efficiently improves the long-range order. So far, we could not observe any influence of the height of the gratings on the ordering.

\subsection{GISAXS on colloidal monolayer}

First we investigate uncoated surface relief gratings. In a second step, the sample was covered with a colloidal monolayer according to Section 3.1 and GISAXS measurements were again performed. The scattering patterns are shown in Fig. 3. The images originate from the same sample: (a) corresponds to the uncoated grid, whereas (b) and (c) correspond to the coated sample. The surface relief gratings are turned around $1.3^{\circ}$ (a), (b) and $-1.3^{\circ}$ (c) with respect to the position where the grating is parallel to the incoming beam. The intensity streak parallel to $q_{Z}$ corresponds to the specularly scattered X-rays (cf. $\mathrm{C}$ in Fig. 3). The peaks angled away from the specular streak arise from the intersection of the Ewald sphere with the grating rods (cf. B in Fig. 3). The central and most intensive peak is the reflected beam (cf. A in Fig. 3). The increase in intensity below $q_{Z} \approx 0.05 \AA$ corresponds to the critical angle of the substrate and the polystyrene beads materials (cf. D in Fig. 3). From the distance of the peaks in $q_{X}$ direction, one can directly extract the grating period of about $860 \mathrm{~nm}$. This corresponds well to the value of $838 \mathrm{~nm}$ obtained by AFM. Comparing the scattering of the uncoated with the coated template, there is a pronounced change in the intensity distribution and the peaks appear to be smeared out in the later case. However, the comparison of the full width at half maximum of the grating peaks along $q_{Y}$ shows almost no difference. This indicates that the correlation length parallel to the surface structures stays unchanged and supports the observation by the light microscope of remarkable long-range order. A more detailed data evaluation is underway.

\subsection{EDXR during self-assembly}

The evaporation of a droplet of colloidal dispersion reveals a pronounced increase in the integrated intensity of the spectra just before complete evaporation of the water. Fig. 4 shows the scattering signal together with the data for the relative humidity at the sample. Obviously, the intensity peak appears simultaneously to the decrease of the humidity. The peak indicates a state including a high order that appears when the last thin layer of liquid is wetting the surface. This is supported by the fact, that several spectra in the region of the peak show reflections due to intermediate well-ordered states. As the thin water film dries completely up the state looses its order partly. It could be understood concerning the following model: When just a small layer of water remains the colloids arrange in an almost perfect tight packed hexagonal lattice due to a combination of convective flow and attractive capillary forces. As the water evaporates completely, the surface tension will brake up the perfect order splitting the crystal in smaller domains [14]. Such domains could also be observed in dried up crystals.
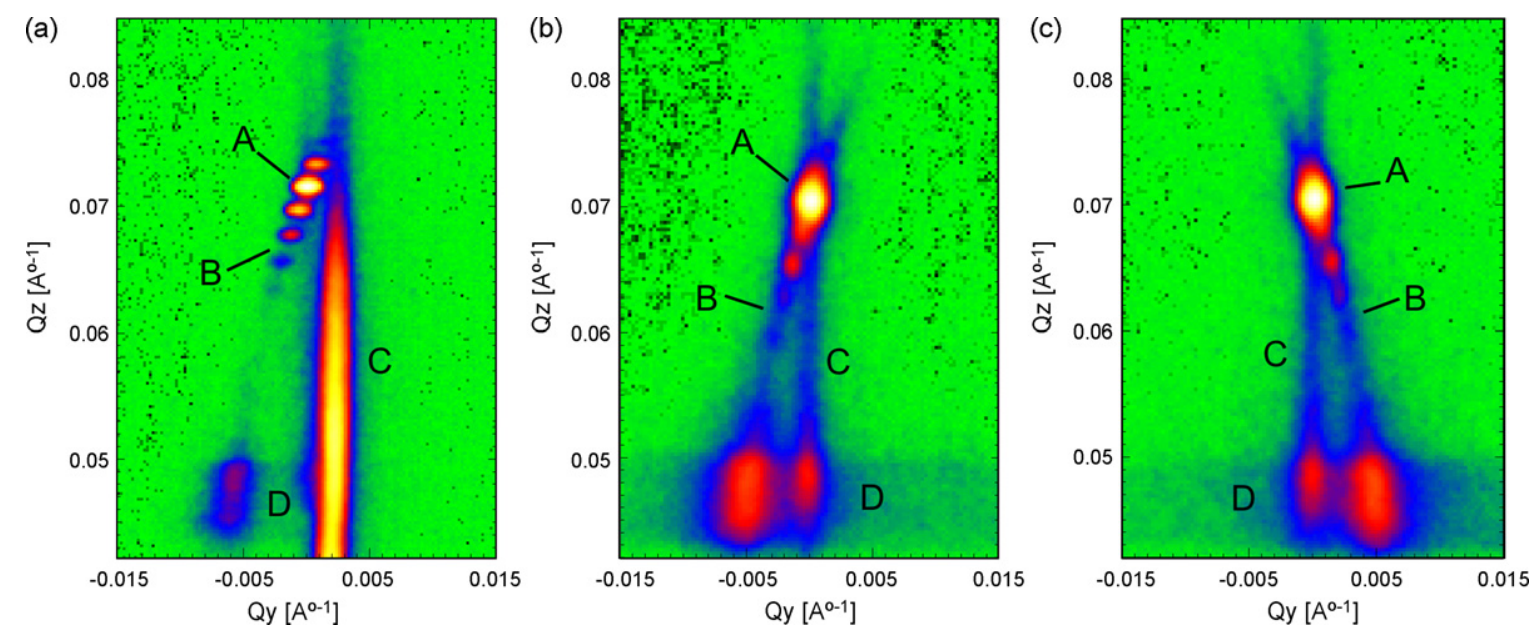

Fig. 3. GISAXS pattern from the same uncoated (a) and coated (b), (c) surface relief grating. The gratings were turned around $1.3^{\circ}$ (a), (b) and $-1.3^{\circ}$ (c) with respect to the position where the grating is parallel to the incoming beam. (A) Reflected beam, (B) grating peaks, (C) specular direction along $q_{Z}$ and (D) Yoneda wing. 


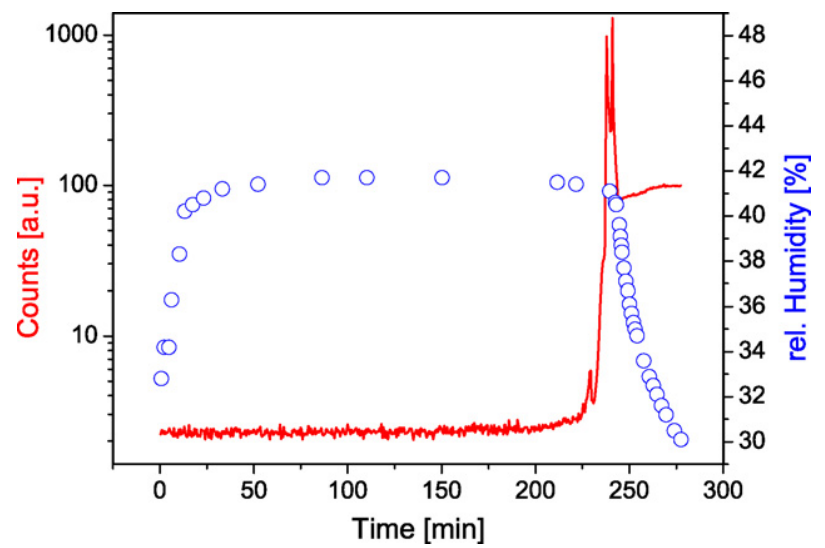

Fig. 4. Integrated scattering intensity of the individual spectra (solid line) and relative humidity (dots) measured during the EDXR measurement: $40 \mathrm{ml}$ colloid dispersion (concentration $0.02 \%$ ) on top of a surface relief grating stayed in the beam until the droplet was evaporated. The enormous increase in intensity appears shortly before the complete evaporation of the droplet.

\section{Conclusion}

Topologically pre-structured templates allow an almost perfect arrangement of monodisperse colloidal spheres onto the surface. We successfully introduced a polymer dye template which can be made in a simple way by a one-step illumination process. Thereby, the topological spacing can be tuned in one (linear grating) as well as in two dimensions (dot grating) to match the size of the colloidal spheres. GISAXS was introductorily used to investigate a colloidal monolayer on a onedimensional topological template. Investigations on coated dot gratings and colloidal multilayer are underway. EDXR enables us to follow in situ the dry up of a droplet of colloidal dispersion. Intermediately, very well ordered structures could be observed. Upon dry up these states are destroyed as small cracks disrupt the larger domains.

\section{Acknowledgements}

The authors would like to thank A. Pucher (University of Potsdam), W. Drube and H. Schulte-Schrepping (Hasylab at DESY), J. Grenzer (FZ Rossendorf), T. Kraus (ETH Zurich) and L. Holitzner (LNS, PSI) for their support and fruitful discussions. AFM measurements have been performed at the PSI SPM Userlab. This work is supported by the Swiss National Funds Grant No. 200021-107796.

\section{References}

[1] J.D. Joannopoulos, P.R. Villeneuve, S. Fan, Nature 386 (1997) 143149.

[2] A. Imhof, D.J. Pine, Nature 389 (1997) 948-951.

[3] Y.A. Vlasov, X.Z. Bo, J.C. Sturm, D.J. Norris, Nature 414 (2001) 289-293.

[4] P. Jiang, J.F. Bertone, K.S. Hwang, V.L. Colvin, Chem. Mater. 11 (1999) 2132-2140.

[5] S. Reculusa, S. Ravaine, Chem. Mater. 15 (2003) 598-605.

[6] A.V. Petukhov, J.H.J. Thijssen, D.C. 't Hart, A. Imhof, A. van Blaaderen, I.P. Dolbnya, A. Snigirev, A. Moussaïd, I. Snigireva, J. Appl. Crystallogr. 39 (2006) 137-144.

[7] P. Muller-Buschbaum, Anal. Bioanal. Chem. 376 (2003) 3-10.

[8] V. Rossi Albertini, B. Paci, A. Generosi, J. Phys. D: Appl. Phys. 39 (2006) R461-R486.

[9] P. Rochon, E. Batalla, A. Natansohn, Appl. Phys. Lett. 66 (1995) 136138.

[10] M. Allard, E.H. Sargent, P.C. Lewis, E. Kumacheva, Adv. Mater. 16 (2004) 1360 .

[11] K. Nagayama, Colloids Surf. A 109 (1996) 363-374.

[12] W. Drube, H. Schulteschrepping, H.G. Schmidt, R. Treusch, G. Materlik, Rev. Sci. Instrum. 66 (1995) 1668-1670.

[13] U. Pietsch, J. Grenzer, T. Geue, F. Neissendorfer, G. Brezsesinski, C. Symietz, H. Mohwald, W. Gudat, Nucl. Instrum. Methods Phys. Res. A 467 (2001) 1077-1080.

[14] A. van Blaaderen, J.P. Hoogenboom, D.L.J. Vossen, A. Yethiraj, A. van der Horst, K. Visscher, M. Dogterom, Faraday Discuss. 123 (2003) $107-119$. 